\title{
Simulation study on nonlinear frequency shift of narrow band whistler-mode waves in a homogeneous magnetic field
}

\author{
Yuto Katoh* and Yoshiharu Omura \\ Research Institute for Sustainable Humanosphere, Kyoto University, Gokasho, Uji, Kyoto 611-0011, Japan
}

(Received December 3, 2005; Revised March 7, 2006; Accepted April 17, 2006; Online published September 29, 2006)

\begin{abstract}
We study frequency variation of a coherent whistler-mode wave in a homogeneous magnetic field by a selfconsistent simulation model. Simulation results show that an injected whistler-mode wave packet grows due to an instability driven by temperature anisotropy and the amplified wave packet triggers emissions with frequency shift during its propagation. We clarify that the resonant currents $J_{E}$ and $J_{B}$ due to the nonlinear wave-particle interaction play significant roles in both wave growth and frequency variation. Based on the simulation results, we show that the range of the frequency shift in a homogeneous system is quantitatively estimated by the trapping frequency $V_{T}$ of trapped electrons; in a case that the original frequency of the wave packet is $0.62 \Omega_{e}$ and $V_{T}=4.05 \times 10^{-2} c$, the lower and upper frequencies are estimated to be $0.565 \Omega_{e}$ and $0.685 \Omega_{e}$, respectively. The results of the present study reveal that the role of nonlinear trapping is significant in the elementary process of VLF triggered emissions in the equatorial region of the magnetosphere.
\end{abstract}

Key words: Nonlinear trapping, whistler-mode waves, cyclotron resonance, particle simulation.

\section{Introduction}

We often realize that the Earth's magnetosphere is a natural laboratory for studying plasma physics. VLF triggered emission is a typical example of experiments in the magnetosphere. Observational results reveal that frequency varying narrow band whistler-mode waves are periodically generated by triggering waves which are emitted from the ground station at high latitude region (Helliwell and Katsufrakis, 1974; Helliwell, 1983). There have been many theoretical and simulation studies investigating generation mechanism of VLF triggered emissions (see reviews by Matsumoto, 1979; Omura et al., 1991). They revealed that nonlinear trapping of resonant electrons produce resonant currents which induce both wave growth and frequency variation of triggered emissions (Vomvoridis and Denavit, 1980; Omura and Matsumoto, 1982, 1985; Omura et al., 1991; Trakhtengerts et al., 2003; Nunn et al., 2003).

The generation and propagation process of whistlermode waves in the Earth's magnetosphere have been recognized as an important research subject in magnetospheric physics (e.g. Bujarbarua et al., 1998; Singh et al., 2004). Results of in situ observations showed that nonlinear processes generating VLF triggered emissions initiate in the equatorial region of the magnetosphere. To investigate the early stage of the triggering mechanism in the equatorial region, Omura and Matsumoto (1985) studied frequency variation of triggered emissions in a homogeneous field as-

*Now at Planetary Plasma and Atmospheric Research Center, Graduate School of Science, Tohoku University, 980-8578, Japan.

Copyright (c) The Society of Geomagnetism and Earth, Planetary and Space Sciences (SGEPSS); The Seismological Society of Japan; The Volcanological Society of Japan; The Geodetic Society of Japan; The Japanese Society for Planetary Sciences; TERRAPUB. suming a coherent whistler-mode wave. They showed that frequency variation of triggered emissions is caused by resonant currents due to resonant electrons trapped by the triggering pulse. The contribution of resonant currents on both wave amplitude and frequency in a uniform magnetic field is described by the following equations (e.g. Nunn, 1974; Omura et al., 1991).

$$
\begin{aligned}
& \left(\frac{\partial}{\partial t}+v_{g} \frac{\partial}{\partial x}\right) B_{W}=-v_{g} \frac{\mu_{0}}{2} J_{E} \\
& \left(\frac{\partial}{\partial t}+v_{g} \frac{\partial}{\partial x}\right) \phi=-v_{g} \frac{\mu_{0}}{2} \frac{J_{B}}{B_{W}}
\end{aligned}
$$

where $J_{E}$ and $J_{B}$ are the components of the transverse resonant currents parallel to the wave electric field $E_{W}$ and to the wave magnetic field $B_{W}$, respectively, and $\phi$ and $v_{g}$ respectively denote the wave phase and the group velocity. As expressed by (1) and (2), the negative $J_{E}$ causes the wave growth and the negative $J_{B}$ induces the rising tone of wave frequency. Omura and Matsumoto (1985) performed a simulation study on the frequency variation of a wave packet in a uniform magnetic field and showed that the frequency rising of a triggered emission occurs after slight decrease of wave frequency due to the positive resonant currents $J_{B}$ formed by trapped electrons. However, since their model treated only a single monochromatic whistler-mode wave, the frequency variation in a homogeneous system should be examined by using a model which can treat a band of waves with a finite wave spectrum.

In this paper we study the triggering mechanism in a homogeneous magnetic field by using one-dimensional Electron Hybrid Model (Katoh et al., 2005b). Electron Hybrid Model has an advantage that wave-particle interaction between triggering wave and resonant electrons are selfconsistently treated. In Section 2, we briefly describe Elec- 
tron Hybrid Model and initial parameters used in the present study. We discuss simulation results in Section 3 and summarize in Section 4.

\section{Simulation Model}

We use Electron Hybrid Model which treats cold electrons as a fluid and hot electrons as particles (Katoh and Omura, 2004; Katoh et al., 2005a). By using the fluid approximation, we can reduce computational costs to solve gyromotions of cold electrons with taking into account the dispersion of cold plasma medium. We assume two electron species as follows. The background cold electrons, we refer as species 1 , are treated as a fluid and their motions are solved by the fluid equation of motion, whereas the electron species 2 is treated as particles including the relativistic effect. The temporal evolution of number density of cold electrons is solved by the continuity equation. Thus nonlinear effects related to the motion of the species 1 is taking into account in the present simulation model. The motion of the species 2 is computed by the BunemanBoris method (Buneman, 1993) while the current density at each grid is computed by standard PIC (Particle-in-Cell) method. In addition, the evolution of electromagnetic fields is directly solved by Maxwell's equations. These features of Electron Hybrid Model enable us to treat wave-particle interaction between plasma waves with a finite bandwidth and energetic electrons with a full distribution function selfconsistently.

We assume that the simulation system is a homogeneous system aligned with the ambient magnetic field direction. In the present study, we treat a whistler-mode wave packet traveling into the simulation system and calculate its propagation by solving Maxwell's equations. To see the fun-

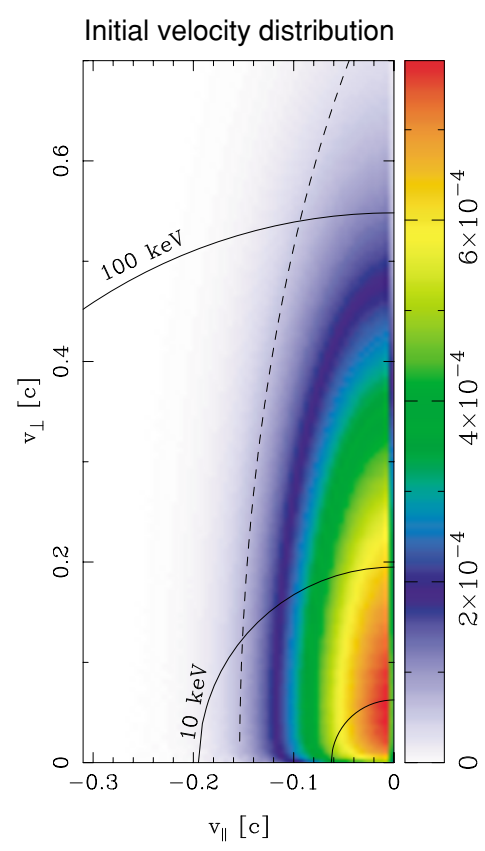

Fig. 1. Initial velocity distribution of the electron species 2. Three solid lines denote constant energy lines of 1, 10, $100 \mathrm{keV}$. Dashed line represents the resonance ellipse of whistler mode wave of frequency of $0.62 \Omega e$. damental physics of triggering mechanism, we treat purely transversal waves by neglecting electrostatic component of electric field. By employing absorbing boundaries (after Umeda et al., 2001) on both edges of the simulation system, we maintain open boundary condition.

We perform three simulation runs using different amplitude of the wave packet $B_{W}$ given in Table 2 . In each simulation run, we assume that the ratio between plasma and gyrofrequency of the electron species $1, \Pi_{e} / \Omega_{e}$, is 2 and that the electron species 2 has a half Maxwellian distribution with a temperature anisotropy as shown in Fig. 1. It has been widely studied that the temperature anisotropy induces an instability generating a narrow band whistler-mode wave (Bujarbarua et al., 1998; Katoh and Omura, 2004). We assume $v_{t h, \|}=0.1 c$ and $v_{t h, \perp}=0.4 c$ where $v_{t h, \|}$ and $v_{t h, \perp}$ represent parallel and perpendicular component of thermal velocity, respectively. Table 2 gives other parameters used in the present study. Under the present parameters, the liner theory suggests that a narrowband whistler-mode wave is excited with the maximum growth rate of $1.12 \times 10^{-3} \Omega_{e}$ at the frequency of $0.62 \Omega e$. By assuming the frequency of the whistler-mode wave packet as $0.62 \Omega e$, we study the growth of the wave packet due to the instability during its propagation in the simulation system.

In this paper, we describe key parameters in real numbers as well as normalized values. By assuming the background magnetic field intensity as $486 \mathrm{nT}$ corresponding to the value at $L=4$, we estimate that the cyclotron frequency of electron is $13.60 \mathrm{kHz}$, cold plasma density is 9.18 /cc, and the frequency of the wave packet is $8.43 \mathrm{kHz}$. Hereinafter we use $B_{0}=486 \mathrm{nT}$ in representing real numbers.

\section{Results and Discussions}

In this paper we mainly discuss results of Run 2, because the fundamental physics appeared in three simulation runs are basically the same.

Spatial distribution of wave spectra at initial stage of Run

Table 1. Initial amplitude of wave packet in each simulation run.

\begin{tabular}{cc}
\hline & $B_{W} / B_{0}$ \\
\hline Run 1 & $4.0 \times 10^{-4}$ \\
Run 2 & $1.6 \times 10^{-3}$ \\
Run 3 & $6.4 \times 10^{-3}$
\end{tabular}

Table 2. Initial parameters used in the present study. Subscripts 1 and 2 refer the component of the electron species 1 and 2, respectively.

$\begin{array}{lr}\text { Plasma frequency of background electron } \Pi_{e} & 2 \Omega_{e} \\ \text { Perpendicular thermal velocity } v_{t h, \perp} & 0.4 \mathrm{c} \\ \text { Parallel thermal velocity } v_{t h, \|} & 0.1 \mathrm{c} \\ \text { Grid spacing } \Delta x & 0.06 \mathrm{c} / \Omega_{e} \\ \text { Time step } \Delta t & 0.01 \Omega_{e}^{-1} \\ \text { Number of grid points } L_{x} & 8192 \\ \text { Density ratio of the electron species } 1 \text { and } 2 & 10^{-3} \\ \text { Number of superparticles in each cell } & 2048 \\ \quad \text { representing the electron species } 2 & \end{array}$




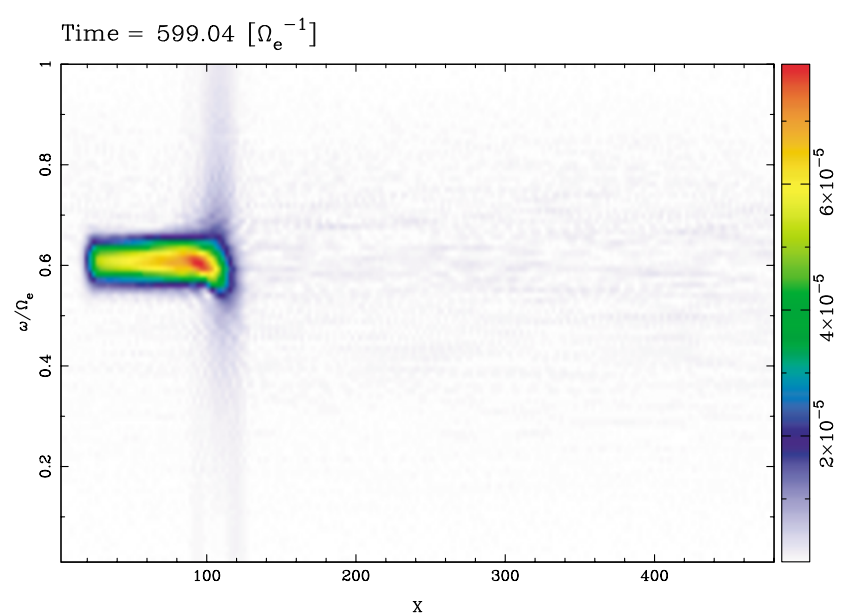

Fig. 2. Spatial distribution of wave spectra at $t=599 \Omega_{e}^{-1}$ in Run 2 .

2 is shown in Fig. 2. The initial amplitude of the whistlermode wave packet corresponds to $778 \mathrm{pT}$. The wave packet interacts continuously with counter-streaming electrons of the species 2 via first order cyclotron resonance. In Fig. 3, we show $v_{\|}-X$ distribution, wave form of wave magnetic field $B_{y}$ and spatial distribution of wave spectrum at $t=$ $2200 \Omega_{e}^{-1}$. The amplitude of the wave packet grows with the growth rate of $39.7 \mathrm{~dB} / \mathrm{s}$ due to the instability driven by the temperature anisotropy, and the emissions are triggered at the downstream of the wave packet. The result of spectrum analysis shows that our self-consistent model successfully reproduces triggered emissions with frequency variation. Figure 4 shows velocity distribution of the electron species 2 at $t=2500 \Omega_{e}^{-1}$; resonant electrons are mainly diffused along the resonance ellipse of frequency of $0.62 \Omega_{e}$ due to the effect of the nonlinear trapping. The effect of the nonlinear trapping also appeared as a streak in $v_{\|}-X$ plot of Fig. 3. The spatial variation of the streak in Fig. 3 shows that the size of a trapping region varies due to the growth of whistler-mode waves.

To assimilate a result of satellite observations, we show wave spectra observed at $X=240 c \Omega_{e}^{-1}$ in Run 2. Figure 5 clearly represents that triggered emissions are generated at the downstream of the injected whistler-mode wave packet of frequency of $0.62 \Omega_{e}$.

\subsection{Formation of resonant currents}

Temporal and spatial variations of resonant currents $J_{E}$ and $J_{B} / B_{W}$ in Run 2 are shown in Fig. 8. The sign of the resonant currents $J_{E}$ and $J_{B}$ is determined by the phase difference $\zeta$ between the phase of the magnetic field component of a whistler-mode wave $\phi$ and gyrophase of a resonant electron $\psi$ as follows:

$$
\begin{gathered}
J_{E}\left\{\begin{array}{l}
>0(0<\zeta<\pi) \\
<0(\pi<\zeta<2 \pi)
\end{array}\right. \\
J_{B}\left\{\begin{array}{l}
>0\left(\frac{\pi}{2}<\zeta<\frac{3 \pi}{2}\right) \\
<0\left(\zeta<\frac{\pi}{2}, \frac{3 \pi}{2}<\zeta\right)
\end{array}\right.
\end{gathered}
$$

where

$$
\zeta=\psi-\phi
$$
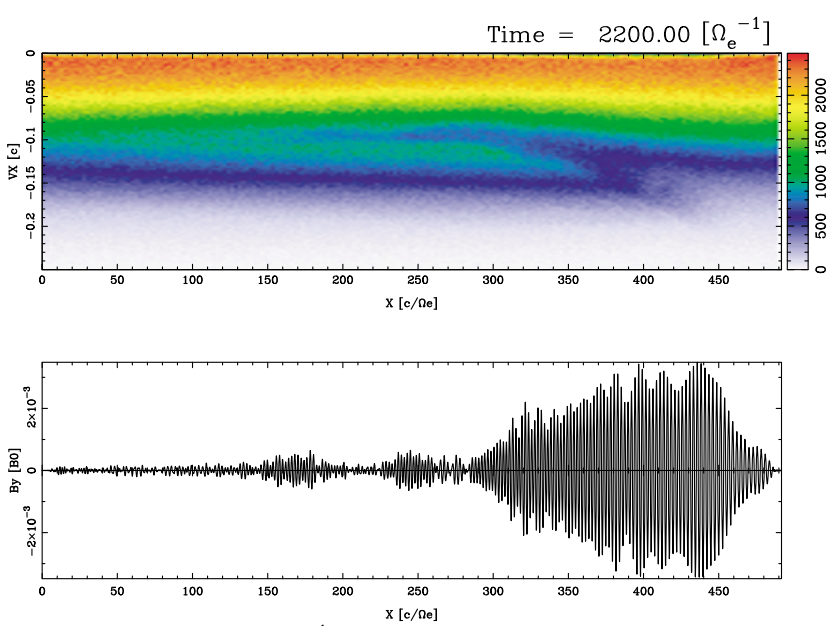

Time $=2190.24\left[\Omega_{\mathrm{e}}^{-1}\right]$

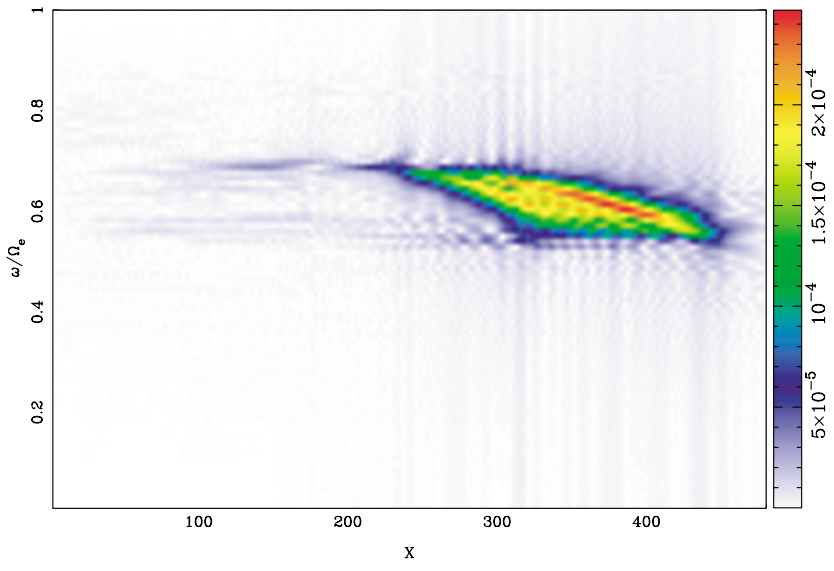

Fig. 3. Simulation result of Run 2. $v_{\|}-X$ distribution (upper panel), wave form of wave magnetic field $B_{y}$ (middle panel) and spatial distribution of wave spectrum (bottom panel) at $t=2200 \Omega_{e}^{-1}$ are shown.

Figure 8 reveals that $J_{E}$ is negative along the propagation path of the triggering wave. This result shows that the resonant current $J_{E}$ formed by the triggering wave contributes to the growth of the triggering wave. In Fig. 3, an amplitude modulation of the whistler-mode waves is appeared around $X=400 \sim 450 c \Omega_{e}^{-1}$. This amplitude modulation should be explained by the oscillation of $J_{E}$ due to the motion of trapped electrons. In the nonlinear trapping process, the trapped electrons oscillate within the trapping region in the phase space with the period of $\omega_{T}^{-1}$ (Matsumoto, 1985), where $\omega_{T}=\sqrt{k v_{\perp 0} q B_{W} / m_{e}}$ is the trapping frequency and $v_{\perp 0}$ is the perpendicular component of the velocity of a trapped electron; we estimate $\omega_{T}$ as $5.33 \times 10^{-2} \Omega_{e}$ (corresponds to $724.88 \mathrm{~Hz}$ ) at $X=440 c \Omega_{e}^{-1}$ in Fig. 3. Since resonant electrons trapped by the triggering wave move toward $-\mathrm{X}$ direction with the resonance velocity $V_{R}=-0.144 c$, spatial scale of the amplitude modulation due to the effect of $J_{E}$ is estimated to $\sim 17 c \Omega_{e}^{-1}$. This estimation of the amplitude modulation caused by the oscillation of $J_{E}$ is consistent with the simulation result as shown in Fig. 3.

Besides, Fig. 8 reveals that $J_{B} / B_{W}$ is positive around the triggering wave packet whereas negative $J_{B} / B_{W}$ is observed at the region of triggered emissions. As expressed by (2), the positive (negative) $J_{B} / B_{W}$ causes decrease (in- 

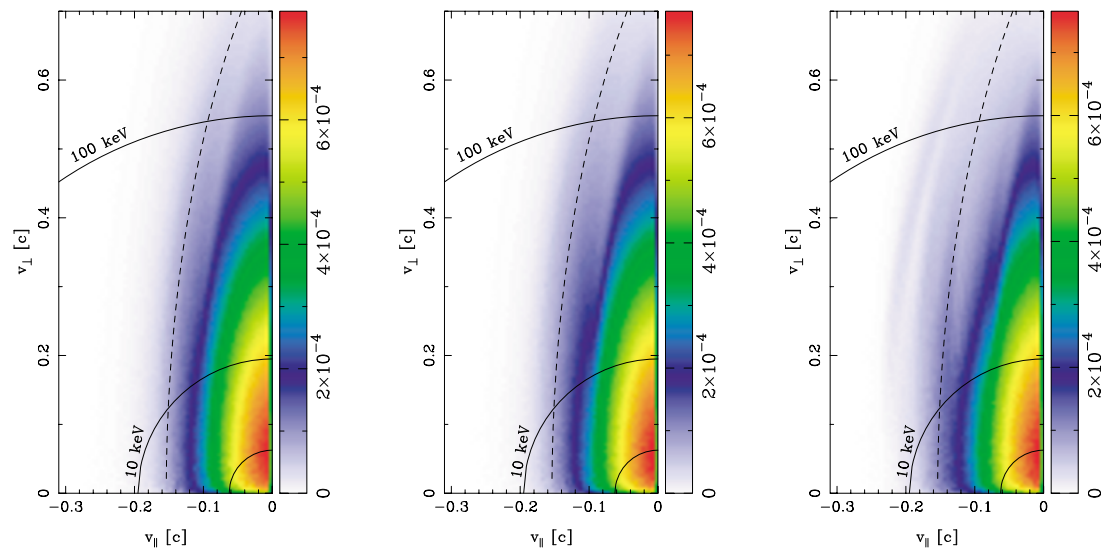

Fig. 4. Velocity distribution of the electron species 2 at $t=2000 \Omega_{e}^{-1}$. Three solid lines denote constant energy lines of $1,10,100 \mathrm{keV}$. Dashed line represents the resonance ellipse of whistler mode wave of frequency of $0.6 \Omega e$.

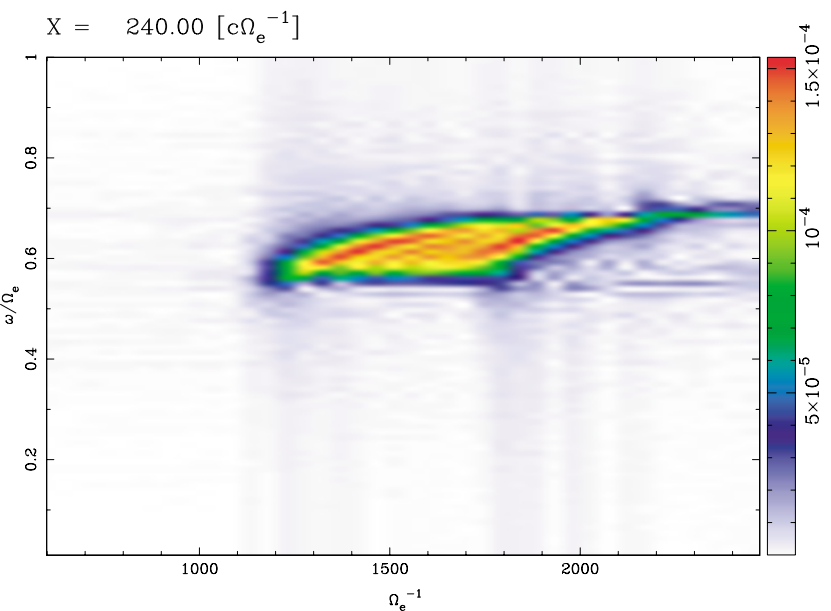

Fig. 5. Wave spectrum observed at $240 c \Omega_{e}^{-1}$ in Run 2. Frequency varying emissions triggered by a whistler mode wave packet $\left(0.62 \Omega_{e}\right)$ are observed.

crease) of the wave frequency. In Fig. 3, we found a slight decrease of wave frequency of the triggering wave during its propagation, which is consistent with the variation of the $J_{B}$ component. It is interesting that the $J_{B} / B_{W}$ component in the region of triggered emissions is mostly negative. This result shows that the frequency of triggered emissions continuously rises due to the effect of the resonant current $J_{B}$. We investigate that what kind of electrons mainly contributed to the formation of resonant currents by examining the distribution of trapped electrons in the phase space. The positive $J_{B} / B_{W}$ observed at the region of the triggering wave is formed by trapped electrons having the phase between $\pi$ and $\frac{3}{2} \pi$, while the negative $J_{B}$ at the region of triggered emissions have the phase between $\frac{3}{2} \pi$ and $2 \pi$.

Let us discuss the formation process of resonant currents in the present simulation. While the triggering whistlermode wave injected into the simulation system grows due to the instability driven by temperature anisotropy, resonant electrons are trapped into the trapping region in $\zeta-v_{\|}$phase space. Since we assumed that the initial velocity distribu-

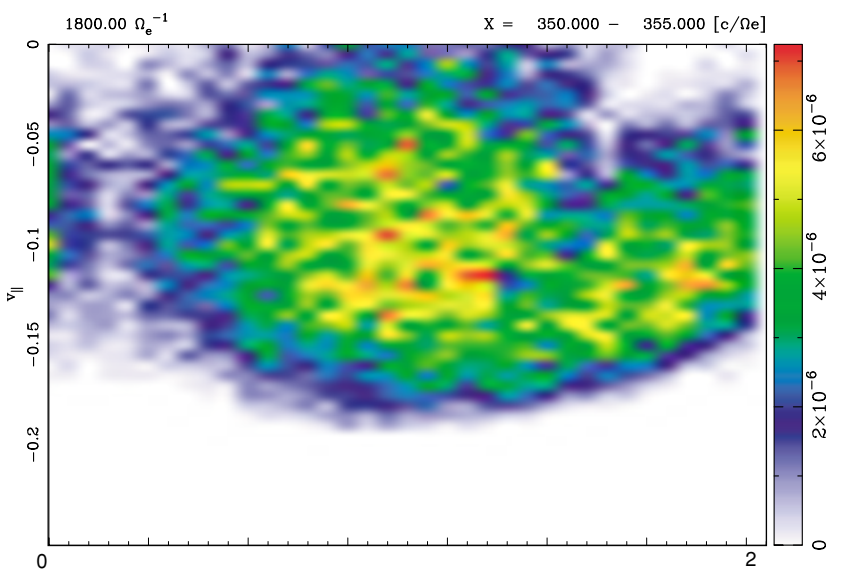

Fig. 6. Distribution of perpendicular component of current density $\left|J_{\perp}\right|$ in the phase space formed by trapped electrons in $X=350 \sim 355 c \Omega_{e}^{-1}$ at $t=1800 \Omega_{e}^{-1}$. Vertical and horizontal axes denote parallel component of particle velocity and phase mismatch between gyrophase and wave magnetic field, respectively.

tion $f_{0}$ of the electron species 2 has $\partial f_{0} / \partial v_{\|}>0$, trapped electrons form a nonuniform distribution in the trapping region resulting in formation of resonant currents. The trapped electrons moving around the phase of $\pi / 2$ contribute the negative $J_{E}$ and the positive $J_{B} / B_{W}$ which cause the growth and the frequency decrease of the triggering wave as we see in the simulation result. In Fig. 6, we show the perpendicular component of the current density distribution $\left|J_{\perp}\right|$ in $X=350.0 \sim 355.0 \mathrm{c} \Omega_{e}^{-1}$ at $t=1800 \Omega_{e}^{-1}$, where the growth of the triggering wave is observed. In calculation of $\left|J_{\perp}\right|$, we summed the contribution from the trapped electrons which are selected with a criterion that $\delta v_{\|}$is greater than $V_{T 0}$, where $V_{T 0}$ is the trapping velocity calculated from the initial wave amplitude of the triggering wave given by $V_{T 0}=2 \sqrt{v_{\perp 0} \Omega_{W 0} / k}$ and $\Omega_{W 0}=q B_{W 0} / m_{e}$. Under the initial condition of Run $2, V_{T 0}$ is estimated to $0.02 c$. Figure 6 clarifies the signature of the spiral motion of trapped electrons.

During the nonlinear interaction process, trapped elec- 
Table 3. Trapping velocity and resultant frequency variation of each simulation run. We assumed $v_{\perp 0}=0.3 c$ in calculation of $V_{T}$.

\begin{tabular}{cccccc}
\hline & $V_{T} / c$ & $\left(V_{R}-V_{T}\right) / c$ & $\left(V_{R}+V_{T}\right) / c$ & $\omega_{\min } / \Omega_{e}$ & $\omega_{\max } / \Omega_{e}$ \\
\hline Run 1 & $2.78 \times 10^{-2}$ & -0.172 & -0.117 & 0.582 & 0.672 \\
Run 2 & $4.05 \times 10^{-2}$ & -0.185 & -0.104 & 0.565 & 0.685 \\
Run 3 & $7.09 \times 10^{-2}$ & -0.215 & -0.073 & 0.530 & 0.745 \\
\hline
\end{tabular}

(a)

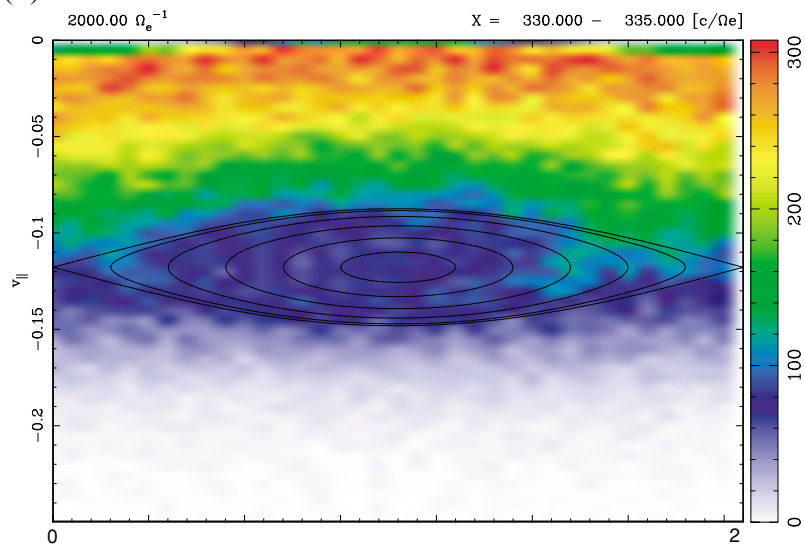

(b)

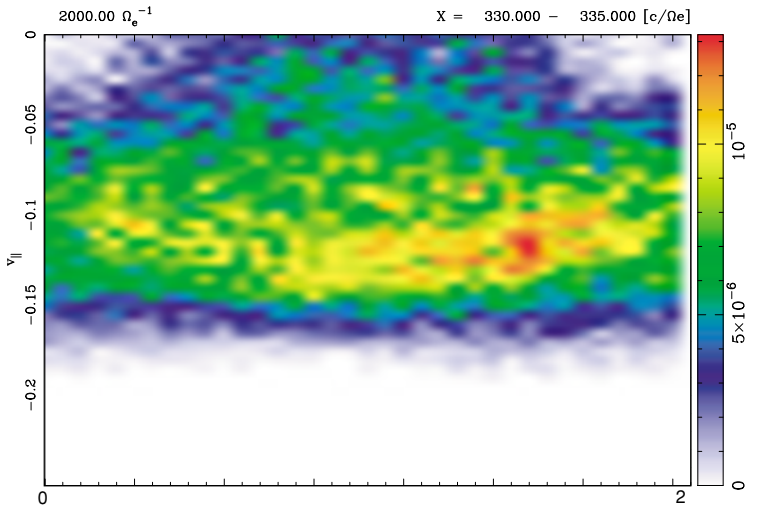

Fig. 7. (a) Phase diagram of electrons and (b) distribution of perpendicular component of current density $\left|J_{\perp}\right|$ formed by trapped electrons in $X=330 \sim 335 c \Omega_{e}^{-1}$ at $t=2000 \Omega_{e}^{-1}$. Format of axes is the same as Fig. 6 .

trons move toward the phase of $2 \pi$. Since the phase of $2 \pi$ in the trapping region corresponds to the saddle point of the nonlinear trapping, motion of trapped electrons located near the separatrix becomes slow as they approach the phase of $2 \pi$. Therefore, trapped electrons located near the separatrix stay around the phase of $2 \pi$ and form negative $J_{B}$ which contribute to the rising tone. However, because of small $J_{B} / B_{W}$ due to the large wave amplitude $B_{W}$, frequency variation should not take place in the region of the triggering wave. During the passage of the counter-streaming triggering wave, trapped electrons pass through the triggering wave and observe the decrease of the wave amplitude. Then the $J_{B} / B_{W}$ due to trapped electrons around $\zeta=2 \pi$ reaches an appropriate value which accounts for the frequency variation. In Fig. 7(a), we show a phase diagram of electrons
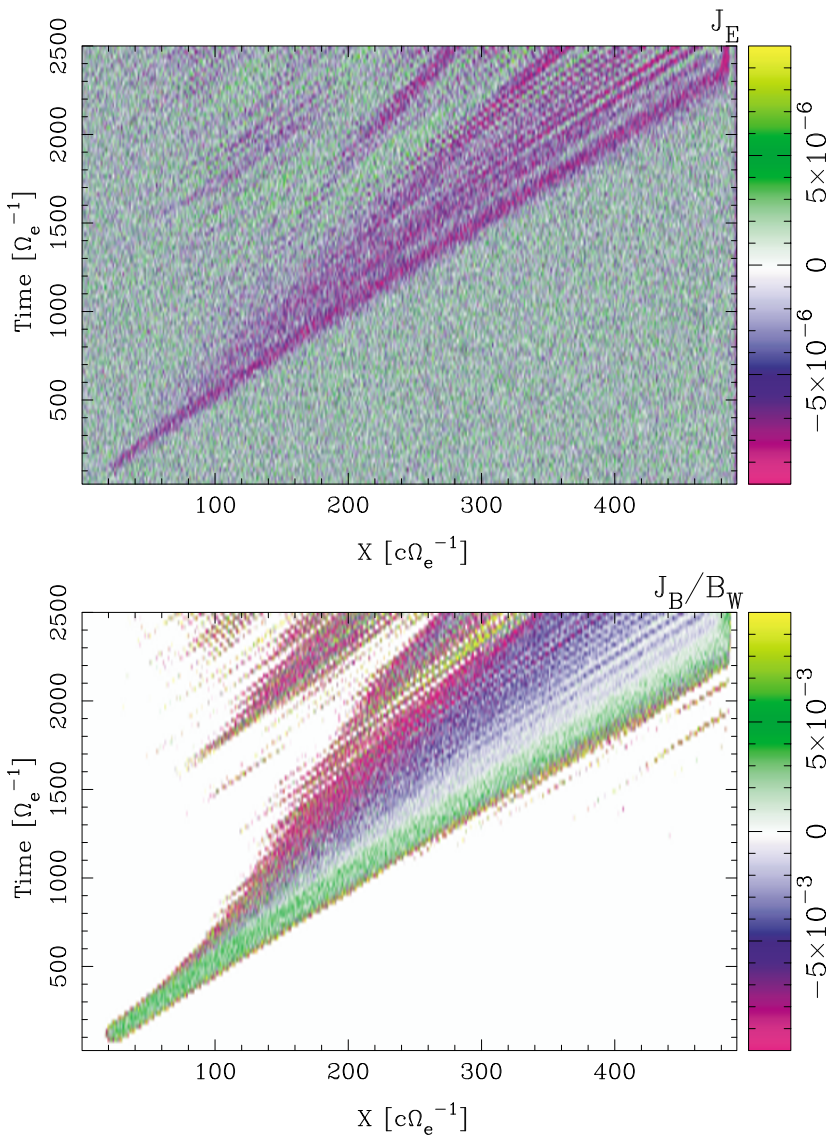

Fig. 8. Resonance currents of $J_{E}$ (upper panel) and $J_{B} / B_{W}$ (lower panel) observed in Run 2. Vertical and horizontal axes represent time step and position in the simulation system, respectively.

in $X=330.0 \sim 335.0 c \Omega_{e}^{-1}$ at $t=2000 \Omega_{e}^{-1}$, where triggered emissions with varying frequency are observed. We also show the perpendicular component of the current density distribution $\left|J_{\perp}\right|$ in the phase space in Fig. 7(b). Figure 7 clarified that resonant electrons are localized around the phase $\sim 2 \pi$ forming strong resonant current $J_{B}$. Moreover, since these trapped electrons also form a negative $J_{E}$, we find wave growth resulting in triggered emissions with rising tone as we find in the simulation result (Fig. 3).

\subsection{Limit of frequency variation}

In Fig. 9, we compare the amount of frequency variation of triggered emissions during $2500 \Omega_{e}^{-1}$ in three simulation runs. The difference of the frequency shift among three simulation runs should be explained by the effect of nonlinear trapping processes as follows. In three simulation runs, we assumed different wave amplitude of the triggering wave packet. Since the motion of trapped electrons in the 


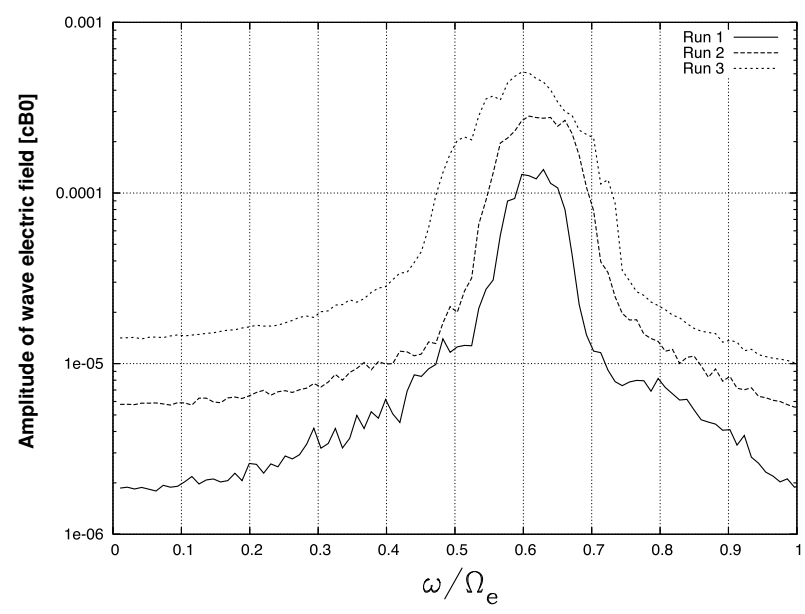

Fig. 9. Wave spectra in Run 1,2 , and 3 at $t=2500 \Omega_{e}^{-1}$.

phase space is examined by the trapping velocity $V_{T}$, strong wave amplitude induces large trapping region given by $V_{R}-V_{T} \leq v_{\|} \leq V_{R}+V_{T}$. Therefore, the effect of resonant currents formed by trapped electrons should be observed within the frequency range from $\omega_{\min }$ to $\omega_{\max }$, where $\omega_{\min }$ and $\omega_{\max }$ represent frequencies of whistler-mode waves satisfying resonance condition with trapped electrons at resonance velocity $V_{R}-V_{T}$ and $V_{R}+V_{T}$, respectively. Table 3 gives $\omega_{\min }, \omega_{\max }$ and other parameters in each simulation run. According to Table 3, the frequency rising in Run 2 is estimated to $6.5 \times 10^{-2} \Omega_{e}$ (corresponds to $884.2 \mathrm{~Hz}$ ). These estimations of the range of the frequency variation are consistent with simulation results (Fig. 9), except $\omega_{\min }$ in Run 3.

\subsection{Self-sustaining process of triggered emissions}

In Run 3, the lower limit of the wave frequency of triggered emissions was less than $0.5 \Omega_{e}$, which is lower than the value of $\omega_{\min }$ given in Table 3 . This difference should be explained by a self-sustaining process caused by the effect of subsequent nonlinear trapping due to the frequency decreased triggering wave. Because the amplitude of the triggering wave packet assumed in Run 3 was highest among three simulation runs, as given in Table 2, the triggering wave in Run 3 trapped a much larger number of resonant electrons than Run 1 and 2 by its large trapping region. Indeed, highly energized electrons appeared in the velocity distribution of Run 3 (Fig. 4). Since larger $\left|v_{\|}\right|$electrons satisfy the resonance condition with lower frequency whistler-mode waves, energized electrons appeared in Run 3 should contribute to the excitation of lower band whistlermode waves. Although the result of Run 3 is not shown in this paper, the frequency of the triggering wave also decreased in Run 3 due to the effect of positive $J_{B}$ and then the resonance velocity $V_{R}$ shifts toward large $\left|v_{\|}\right|$. The change of $V_{R}$ results in the shift of trapping region in the phase space and induces shift of $\omega_{\min }$ of triggered emissions as seen in Run 3.

\section{Summary}

We studied frequency variation of triggered emissions in a homogeneous magnetic field by using one-dimensional
Electron Hybrid Code. Based on the simulation results, we clarified that the resonant currents of trapped electrons play significant roles in both wave growth and frequency variation of triggered emissions. Our model successfully reproduced that the injected whistler-mode wave packet grows due to the instability driven by a temperature anisotropy and the amplified wave packet triggers emissions with a frequency shift during its propagation in a uniform magnetic field. Simulation results were consistent with a model that the wave growth and the frequency variation of triggered emissions are explained by the contribution of resonant currents $J_{E}$ and $J_{B}$. Negative $J_{E}$ and $J_{B}$ respectively cause growth and frequency rising of waves whereas positive $J_{E}$ and $J_{B}$ induce dumping and frequency decrease, respectively.

The formation process of resonant currents and generation process of triggered emissions in the present simulation is summarized as follows:

1) The triggering whistler-mode wave injected into the simulation system grows due to the instability driven by temperature anisotropy.

2) Resonant electrons are trapped into the trapping region of the amplified triggering wave around the phase of $\pi / 2$ in the phase space forming negative $J_{E}$ and positive $J_{B}$ which cause the growth and frequency decrease of the wave.

3) Trapped electrons move toward the phase of $2 \pi$ which corresponds to the saddle point of the nonlinear trapping.

4) While trapped electrons observe a decrease of wave amplitude due to the passage of the triggering wave, the trapped electrons around $\zeta=2 \pi$ form negative $J_{B} / B_{W}$ and negative $J_{E}$ which contribute to the rising tone and the growth of the triggered emissions.

Besides, based on the simulation results, we clarified that the range of the frequency shift is quantitatively estimated by both trapping velocity $V_{T}$ and resonance velocity $V_{R}$ as given by Table 3 .

The results of the present study also clarified that Electron Hybrid Code is a useful tool to investigate the waveparticle interaction between energetic electrons and waves with a finite bandwidth propagating in a cold plasma medium. The physics of the triggered emissions has been investigated by previous simulation studies using VHS code (Nunn et al., 2003, 2005), LTS code (Vomvoridis and Denavit, 1980; Omura and Matsumoto, 1982, 1985) and full particle code (Matsumoto et al., 1980; Hashimoto et al., 1983). We might add the present simulation model to the above list. Electron Hybrid Code has advantages in treatment of electromagnetic fields and full distribution function of energetic particles. While a Vlasov code is a suitable method to investigate the wave-particle interaction, calculation with enough resolution for the velocity space in a large simulation system will costs as large or larger than the present simulation model.

The physics discussed in the present study corresponds to the elementary process of the generation mechanism of VLF triggered emissions in the equatorial region of the Earth's magnetosphere. In the real magnetosphere, there 
exists an inhomogeneity of geomagnetic field, which significantly changes the nonlinear trapping process of resonant electrons. It is necessary to include the effect of the inhomogeneity so as to compare simulations with observational results. Although many theoretical and simulation studies have tried to investigate the triggering mechanism including the effect of an inhomogeneity, detailed physics remains unsolved because of its complexity of nonlinear wave-particle interactions. Such works are important in future studies, and we are now preparing a new simulation study by using Electron Hybrid Code with an inhomogeneity of geomagnetic field. These results will be presented in the near future.

Acknowledgments. Computation in the present study was performed with the KDK system of Research Institute for Sustainable Humanosphere (RISH) at Kyoto University as a collaborative research project. This work was partially supported by Kyoto University Active Geosphere Investigations for the 21 st Century Centers of Excellence Program (KAGI21) and Grant-in-Aid 17340146 and 17GS0208 of the Ministry of Education, Science, Sports and Culture of Japan. Y.K. is supported by a research fellowship of the Japan Society for the Promotion of Science for Young Scientists.

\section{References}

Bujarbarua, S., M. Nambu, B. J. Saikia, M. Eda, and J. I. Sakai, Numerical simulation and theory of generation of electromagnetic waves in the presence of whistler turbulence, Phys. Plasmas, 5(6), 2244-2251, 1998.

Buneman, O., TRISTAN, Computer Space Plasma Physics: Simulation Techniques and Softwares, edited by H. Matsumoto and Y. Omura, pp. 67-84, Terra Scientific Publishing Company, Tokyo, Japan, 1993.

Hashimoto, K., H. Matsumoto, Y. Serizawa, and I. Kimura, Computer simulation of whistler mode wave-particle interactions using a freeboundary encounter model, J. Geophys. Res., 88, 3072-3078, 1983.

Helliwell, R. A., Controlled stimulation of VLF emissions from Siple station, Antarctica, Radio Sci., 18, 801-814, 1983.

Helliwell, R. A. and J. P. Katsufrakis, VLF wave injection into the magnetosphere from Siple station, Antarctica, J. Geophys. Res., 79, 25112518, 1974.

Katoh, Y. and Y. Omura, Acceleration of relativistic electrons due to resonant scattering by whistler mode waves generated by temperature anisotropy in the inner magnetosphere, J. Geophys. Res., 109, A12214, doi:10.1029/2004JA010654, 2004.

Katoh, Y., T. Ono, and M. Iizima, Numerical Simulation of resonant scat- tering of energetic electrons in the outer radiation belt, Earth Planets Space, 57, 117-124, 2005a.

Katoh, Y., T. Ono, and M. Iizima, A numerical study on the resonant scattering process of relativistic electrons via whistler-mode waves in the outer radiation belt, in The Inner Magnetosphere: Physics and Modeling, edited by T. Pulkkinen, R. H. W. Friedel, and N. Tsyganenko, Geophysical Monograph Series, Vol. 155, p. 33-39, 2005 b.

Matsumoto, H., Nonlinear whistler-mode interaction and triggered emissions in the magnetosphere: a review, in Wave Instabilities in Space Plasma, edited by P. J. Palmadesso and K. Papadopulos, pp. 163-190, D. Reidel Pub. Co., 1979.

Matsumoto, H., Coherent nonlinear effects on electromagnetic waveparticle interactions, Space Science Reviews, 42, 429-448, 1985.

Matsumoto, H., K. Hashimoto, and I. Kimura, Dependence of coherent nonlinear whistler interaction on wave amplitude, J. Geophys. Res., $\mathbf{8 5}$, 644-652, 1980.

Nunn, D., A self-consistent theory of triggered VLF emissions, Planet. Space Sci., 22, 349-378, 1974.

Nunn, D., A. Demekhov, V. Trakhtengerts, and M. J. Rycroft, VLF emission triggering by a highly anisotropic energetic electron plasma, $A n$ nales Geophysicae, 21, 481-492, 2003.

Nunn, D., M. J. Rycroft, and V. Trakhtengerts, A parametric study of the numerical simulations of triggered VLF emissions, Annales Geophysicae, 23, 3655-3666, 2005.

Omura, Y. and H. Matsumoto, Computer simulations of basic processes of coherent whistler wave-particle interactions in the magnetosphere, $J$. Geophys. Res., 87(A6), 4435-4444, 1982.

Omura, Y. and H. Matsumoto, Simulation study of frequency variations of VLF triggered emissions in a homogeneous field, J. Geomag. Geoelectr., 37, 829-837, 1985.

Omura, Y., D. Nunn, H. Matsumoto, and M. J. Rycroft, A review of observational, theoretical and numerical studies of VLF triggered emissions, J. Atmos. Terr. Phys., 53, 351-368, 1991.

Singh, K., R. P. Singh, and O. E. Ferencz, Simulation of whistler mode propagation for low latitude stations, Earth Planets Space, 56, 979-987, 2004.

Trakhtengerts, V. Y., A. G. Demekhov, Y. Hobara, and M. Hayakawa, Phase-bunching effects in triggered VLF emissions: Antenna effect, $J$. Geophys. Res., 108(A4), 1160, doi:10.1029/2002JA009415, 2003.

Umeda, T., Y. Omura, and H. Matsumoto, An improved masking method for absorbing boundaries in electromagnetic particle simulations, Comput. Phys. Commun., 137, 286-299, 2001.

Vomvoridis, J. L. and J. Denavit, Nonlinear evolution of a monochromatic whistler wave in a nonuniform magnetic field, Phys. Fluids, 23, 174$183,1980$.

Y. Katoh (e-mail: yuto@pparc.geophys.tohoku.ac.jp) and Y. Omura 